BULL. AUSTRAL. MATH. SOC.

VOL. $27(1983), 91-105$.

\title{
REAL HARDY SPACES OF AN ANNULUS
}

\author{
HWAI-CHIUAN WANG
}

In this article we use real methods to study the Dirichlet and the boundary value problems of an annulus. Then we establish various properties of real Hardy spaces on the annulus, and give some applications.

\section{Introduction}

Fix $r_{0}, 0<r_{0}<1$. Let $\boldsymbol{C}$ be the complex numbers, and $A=\left\{z \in \mathbb{C}: r_{0}<|z|<1\right\}$ be an annulus. The boundary of $A$ consists of two pieces $\Gamma_{0}=\left\{z \in \mathbb{C}:|z|=r_{0}\right\}$, and $\Gamma_{1}=\{z \in \mathbf{C}:|z|=I\}$. Write $\Gamma=\Gamma_{0} \cup \Gamma_{1}$.

In this article, we first study the Dirichlet and the boundary value problems on the annulus $A$. Then we shall construct real Hardy spaces $h^{p}(A)$. We also study the relationships between $h^{p}(A)$ and some important operators such as the Poisson kernel, the conjugate Poisson kernel and the Hilbert transforms. Two related works should be mentioned. The first is Sarason's monograph [4], where Hardy spaces have been studied through complex methods, and then applied to study invariant theory. The second is Goldberg [3], who studied real Hardy spaces on a strip $\{z \in \mathbf{C}: 0<\operatorname{Im} z<1\}$. It is noted that a Hardy space of the annulus does not go directly over to a Hardy space of the strip since circles centered at 0 do not go over into lines parallel to the $\approx$-axis.

Received 14 September 1982. The author wishes to express his gratitude to $R$. Temam for his talks drawing his attention to this problem studied in this paper. 
Actually the Cayley transform (or its inverse) takes these lines into circles tangent to the unit circle at $t t \in$ point -1 . Finally we give some applications to plasma physics.

\section{The Poisson kernel, the conjugate Poisson kernel, and the Hilbert transform}

To derive the Poisson kernel on the annulus $A$, we start with the closed unit disk $D$ minus the points 1 and -1 , apply a suitable conformal mapping onto the upper half plane and then was another conformal mapping onto the strip $B=\{z \in C: 0<\operatorname{Im} z<1\}$. Finally, we roll $B$ up to $A$.

Following Sarason [4], p. 22, consider the holomorphic function

$$
F(t, r)=\frac{1}{2 q_{0}} \tanh \left|-\frac{\pi t}{2 q_{0}}+i\left(\frac{\pi}{4}+\frac{\pi}{2 q_{0}} \log \frac{r}{r_{0}^{\frac{1}{2}}}\right)\right|
$$

where $q_{0}=-\log r_{0}, 0<r_{0}<r<1, t \in \mathbb{R}$, the real numbers. The imaginary part $P(t, r)$ and the real part $Q(t, r)$ of $F(t, r)$ are as follows :

$$
\begin{aligned}
& P(r, t)=\frac{\left(1 / 2 q_{0}\right) \cos \left[\left(\pi / q_{0}\right) \log \left(r / r_{0}^{\frac{1}{2}}\right)\right]}{\cosh \left(\pi t / q_{0}\right)-\sin \left[\left(\pi / q_{0}\right) \log \left(r / r_{0}^{\frac{1}{2}}\right)\right]}, \\
& Q(t, r)=\frac{-\sinh \left(\pi t / q_{0}\right)}{\cosh \left(\pi t / q_{0}\right)-\sin \left[\left(\pi / q_{0}\right) \log \left(r / r_{0}^{\frac{1}{2}}\right)\right]} .
\end{aligned}
$$

Thus $P$ and $Q$ are harmonic functions. Furthermore

$$
\int_{-\infty}^{\infty} P(t, r) d t+\int_{-\infty}^{\infty} P\left(t, \frac{r_{0}}{r}\right) d t=1 \text { for } r_{0}<r<1,
$$

$$
|P(t+2 \pi k, r)| \leq \min \left\{\frac{c}{k^{4}}, \frac{c \cos \left[\left(\pi / q_{0}\right) \log \left(r / r_{0}^{\frac{1}{2}}\right)\right]}{|t|^{4}}\right\}
$$

for large $k,|t| \leq \pi$.

Proof of (2.3). Sirice 


$$
P(t+2 \pi k, r)=\frac{\left(1 / 2 q_{0}\right) \cos \left(\left(\pi / q_{0}\right) \log \left(r / r_{0}^{\frac{1}{2}}\right)\right)}{\cosh \left(\pi / q_{0}\right)(t+2 \pi k)-\sin \left(\left(\pi / q_{0}\right) \log \left(r / r_{0}^{\frac{1}{2}}\right)\right]},
$$

we have

$$
\begin{aligned}
|P(t+2 \pi k, r)| & \leq \frac{1}{\cosh \left(\pi / q_{0}\right)(t+2 \pi k)-1} \\
& \leq \frac{c}{k^{4}} \text { for }|t| \leq \pi \text { and large } k,
\end{aligned}
$$

or

$$
\begin{aligned}
|P(t+2 \pi k, r)| & \leq \frac{\left(1 / 2 q_{0}\right) \cos \left(\left(\pi / q_{0}\right) \log \left(r / r_{0}^{\frac{1}{2}}\right)\right]}{\cosh \left(\pi / q_{0}\right)(t+2 \pi k)-1} \\
& \leq \frac{\cos \left(\left(\pi / q_{0}\right) \log \left(r / r_{0}^{\frac{1}{2}}\right)\right]}{(t+2 \pi k)^{4}} \\
& \leq \frac{\cos \left(\left(\pi / q_{0}\right) \log \left(r / r_{0}^{\frac{1}{2}}\right)\right)}{|t|^{4}} \text { for large } k .
\end{aligned}
$$

Similar arguments reveal

$$
|P(t+2 \pi k, r)| \leq \frac{c}{|t|} \text { for }|t| \leq \pi \text { and large } k .
$$

By (2.3) we have

(2.4) $|P(t+2 \pi k, r)| \leq \frac{\cos \left(\left(\pi / q_{0}\right) \log \left(r / r_{0}^{\frac{3}{2}}\right)\right]}{k^{2}|t|^{2}}$ for $|t| \leq \pi$ and large $k$,

$$
\left|\frac{\partial^{2} P(t+2 \pi k, r)}{\partial t^{2}}\right| \leq \frac{c}{k^{2}} \text { for }|t| \leq \pi \text { and large } k \text {, }
$$

$$
\left|\frac{\partial^{2} P(t+2 \pi k, r)}{\partial r^{2}}\right| \leq \frac{c}{k^{2}} \text { for }|t| \leq \pi \text { and large } k \text {. }
$$

For $|t| \leq \pi, \quad r_{0}<r<1$, define

$$
p(t, r)=\sum_{-\infty}^{\infty} P(t+2 \pi k, r)
$$

By inequalities (2.1) $-(2.6)$, we obtain 


$$
\begin{gathered}
p(t, r) \text { is harmonic on } A, \\
p(t, r)>0 \text { for } r_{0}<r<1,|t| \leq \pi, \\
\frac{1}{2 \pi} \int_{0}^{2 \pi} p(t, r) d t+\frac{1}{2 \pi} \int_{0}^{2 \pi} p\left(t, \frac{r_{0}}{r}\right) d t=1,
\end{gathered}
$$

(2.10) $\sup _{r_{0}<r<1}\left|p_{r} * f(t)\right| \leq c M f(t)$ for $f \in L^{1}(\Gamma)$ where $p_{p}(t)=p(t, r)$ and $M f$ is the Hardy-Littlewood maximal function of $f$,

(2.11) for any $\delta, 0<\delta<\pi$, we have

$$
\lim _{r \rightarrow 1} \frac{1}{2 \pi} \int_{|t| \geq \delta} p_{r}(t) d t=\lim _{r \rightarrow r_{0}} \frac{1}{2 \pi} \int_{|t| \geq \delta} p_{r_{0} / r}(t) d t=0 .
$$

Proof of (2.11). By inequality (2.4),

$\left|p_{p}(t)\right| \leq \frac{\cos \left(\left(\pi / q_{0}\right) \log \left(r / r_{0}^{\frac{1}{2}}\right)\right)}{|t|^{2}} \rightarrow 0$ almost everywhere as $r \rightarrow 1$.

Similarly $p_{r_{0} / r}(t) \rightarrow 0$ almost everywhere as $r \rightarrow r_{0}$.

Using $\left|p_{r}(t)\right| \leq c /|t|^{2}$ on $\delta \leq|t| \leq \pi$ and the Lebesgue dominated convergence theorem,

$$
\lim _{p \rightarrow 1} \frac{1}{2 \pi} \int_{|t| \geq \delta} p_{p}(t) d t=\lim _{r \rightarrow p_{0}} \frac{1}{2 \pi} \int_{|t| \geq \delta} p_{r}(t) d t=0 .
$$

This proves the assertion.

Set $p_{r}^{1}(t)=p_{r}(t)$ and $p_{r}^{0}(t)=p_{r_{0} / r}(t)$.

THEOREM 1. Let $f_{0} \in C\left(\Gamma_{0}\right), f_{1} \in C\left(\Gamma_{1}\right)$. Then

$$
u(t, r)=p_{r}^{0} * f_{0}^{1}(t)+p_{r}^{1} * f_{1}(t)
$$

solves the Dimichlet problem on $A$.

Proof. By $(2.7), p(t, r)$ is harmonic on $A$. Write 


$$
\begin{aligned}
& u(t, r)-f_{0}(t)-f_{1}(t) \\
& \left.=\int p_{r}^{0} * f_{0}(t)-f_{0}(t)\right)+\left(p_{r}^{1} * f_{1}(t)-f_{1}(t)\right) \\
& =\frac{1}{2 \pi} \int p_{r}^{0}(u)\left|f_{0}(t-u)-f_{0}(t)\right| d u-\frac{1}{2 \pi} \int p_{r}^{1}(u) f_{0}(t) d u \\
& \quad+\frac{1}{2 \pi} \int p_{r}^{1}(u)\left|f_{1}(t-u)-f_{1}(t)\right| d u-\frac{1}{2 \pi} \int p_{r}^{0}(u) f_{1}(t) d u .
\end{aligned}
$$

For $\varepsilon>0$ take $\delta>0$ such that

$$
\left\|f_{0}(t-u)-f_{0}(t)\right\|_{\infty}<\frac{\varepsilon}{c+1} \text { whenever }|u| \leq \delta
$$

But

$$
\begin{aligned}
\left|\frac{1}{2 \pi} \int p_{r}^{0}(u)\right| f_{0}(t-u)-f_{0}(t)|d u| & \leq \frac{I}{2 \pi} \int p_{r}^{0}(u)\left|f_{0}(t-u)-f_{0}(t)\right| d u \\
& =\frac{1}{2 \pi} \cdot 2\left\|f_{0}\right\|_{\infty} \int_{|u|>\delta} p_{r}^{0}(u) d u \\
& \quad+\frac{c}{2 \pi} \int_{|u| \leq \delta}\left|f_{0}(t-u)-f_{0}(t)\right| d u \\
& <c \int_{|u|>\delta} p_{r}^{0}(u) d u+\varepsilon \\
& \rightarrow \varepsilon \text { as } r \rightarrow r_{0},
\end{aligned}
$$$$
\left|\frac{1}{2 \pi} \int p_{r}^{l}(u) f_{0}(t) d u\right| \leq \frac{1}{2 \pi}\left\|f_{0}\right\|_{\infty} c \cos \left(\frac{\pi}{q_{0}} \log \frac{r}{r_{0}^{\frac{3}{2}}}\right) \rightarrow 0 \text { as } r \rightarrow 1 \text {. }
$$

The estimates of the third and the fourth terms are similar. Hence

$$
\lim _{r \rightarrow 1} u(t, r)=f_{1}(t) \text { and } \lim _{t \rightarrow r_{0}} u(t, r)=f_{0}(t) \text {. }
$$

This completes the proof.

We come to the boundary value problem on $A$.

THEOREM 2. SUppose $u$ is harmonic on $A, 1<p<\infty$. Then

$$
\sup _{r_{0}<r<1} \frac{1}{2 \pi} \int_{0}^{2 \pi}|u(t, r)|^{p} d t<\infty
$$

if and only if

$$
u(t, r)=p_{r}^{0} * f_{0}(t)+p_{r}^{I} * f_{1}(t)
$$


96

Hwai-chivan Wang

for some $f_{0} \in L^{p}\left(\Gamma_{0}\right)$ and $f_{1} \in L^{p}\left(\Gamma_{1}\right)$.

Proof. It suffices to prove the only if part.

Let

$$
\sup _{r_{0}<r<1} \frac{1}{2 \pi} \int_{0}^{2}|u(t, r)|^{p} d t<\infty
$$

By the Banach-Alauglu theorem, there exists $f_{0} \in L^{p}\left(\Gamma_{0}\right), f_{1} \in L^{p}\left(\Gamma_{1}\right)$ such that

$$
\begin{aligned}
& u\left(t, r_{k}\right) \rightarrow f_{0}(t) \text { as } r_{k} \rightarrow 1, \\
& u\left(t, s_{k}\right) \rightarrow f_{1}(t) \text { as } s_{k} \rightarrow r_{0},
\end{aligned}
$$

in the $w^{*}$-topology,

$$
\text { Note that } g_{k}(t)=u\left(t, r_{k}\right) \in C\left(\Gamma_{0}\right) \text { and } h_{k}(t)=u\left(t, s_{k}\right) \in C\left(\Gamma_{1}\right) \text {. }
$$

By Theorem 1,

$$
\int u\left(x, r_{k}\right) p_{r}^{1}(t-x) d x+\int u\left(x, s_{k}\right) p_{r}^{0}(t-x) d x \rightarrow\left\{\begin{array}{lll}
u\left(t, r_{k}\right) & \text { as } & r \rightarrow 1, \\
u\left(t, s_{k}\right) & \text { as } r \rightarrow r_{0}
\end{array} .\right.
$$

By the uniqueness of harmonic functions on $A$, and

$$
u\left(t, \frac{(1-r) s_{k}+\left(r-r_{0}\right) r_{k}}{1-r_{0}}\right) \rightarrow\left\{\begin{array}{lll}
u\left(t, r_{k}\right) & \text { as } & r \rightarrow 1, \\
u\left(t, s_{k}\right) & \text { as } & r \rightarrow r_{0}
\end{array},\right.
$$

we obtain

$$
u\left(t, \frac{(1-r) s_{k}+\left(r-r_{0}\right) r_{k}}{1-r_{0}}\right)=\int u\left(x, r_{k}\right) p_{r}^{1}(t-x) d x+\int u\left(x, s_{k}\right) p_{r}^{0}(t-x) d x
$$

letting $s_{k} \rightarrow r_{0}$ and $r_{k} \rightarrow 1$, we have

$$
\begin{aligned}
u(t, r) & =\int f_{1}(x) p_{r}^{1}(t-x) d x+\int f_{0}(x) p_{p}^{0}(t-x) d x \\
& =p_{r}^{0} * f_{0}(t)+p_{r}^{1} * f_{1}(t) .
\end{aligned}
$$

This completes the proof.

https://doi.org/10.1017/S0004972700011515 Published online by Cambridge University Press 
Theorem 2 can be extended to the Sobolev space $L_{k}^{p}(\Gamma)$ of order $k$.

THEOREM 3. For $1 \leq p<\infty, k$ a positive integer.

(a) For $f_{0} \in L_{k}^{p}\left(\Gamma_{0}\right), f_{1} \in L_{k}^{p}\left(\Gamma_{1}\right)$, let

$$
u(t, r)=f_{0} * p_{r}^{0}(t)+f_{1} * p_{r}^{1}(t) .
$$

Then $u(t, r)$ is harmonic on $A$,

$$
\begin{aligned}
\lim _{r \rightarrow r_{0}} u(t, r) & =f_{0}(t) \text { almost everywhere, } \\
\lim _{r \rightarrow 1} u(t, r) & =f_{1}(t) \text { almost everywhere, } \\
\|u(t, r)\|_{L_{k}^{p}(\Gamma)} & \leq\left\|f_{0}\right\|_{L_{k}^{p}\left(\Gamma_{0}\right)}+\left\|f_{1}\right\|_{L_{k}^{p}\left(\Gamma_{1}\right)},
\end{aligned}
$$

for all $r, r_{0}<r<1$.

(b) Suppose $u(t, r)$ is harmonic on $A$. Assume there is $c>0$, for some $p, 1 \leq p \leq \infty$,

$$
\|u(t, r)\|_{L_{k}^{p}(\Gamma)} \leq c \text { for all } r, r_{0}<r<1 .
$$

Then

(i) for $1<p<\infty$,

$$
u(t, r)=f_{0} * p_{r}^{0}(t)+f_{1} * p_{r}^{1}(t)
$$

for some $f_{0} \in L_{k}^{p}\left(\Gamma_{0}\right), f_{1} \in L_{k}^{p}\left(\Gamma_{1}\right)$;

(ii) for $p=1$ : if $\left\{f_{r}\right\}$ is cauchy in $L^{1}$-norm as $r \rightarrow 1$ and $r \rightarrow r_{0}$ where $f_{r}(t)=u(t, r)$, then

$$
u(t, r)=f_{0} * p_{r}^{0}(t)+f_{1} * p_{p}^{1}(t)
$$

for some $f_{0} \in L_{k}^{1}\left(\Gamma_{0}\right)$ and $f_{1} \in L_{k}^{l}\left(\Gamma_{1}\right)$.

Proof. It suffices to prove (b) (i). By Theorem 2, take 
$f_{0}^{\alpha} \in L^{p}\left(\Gamma_{0}\right), f_{1}^{\alpha} \in L^{p}\left(\Gamma_{1}\right)$ with

$$
\frac{\partial^{\alpha}}{\partial t^{\alpha}}\left(p_{r}^{0} * f_{0}(t)\right)=p_{r}^{0} * f_{0}^{\alpha}
$$

and

$$
\frac{\partial^{\alpha}}{\partial t^{\alpha}}\left(p_{r}^{1} * f_{1}(t)\right)=p_{r}^{1} * f_{1}^{\alpha}(t)
$$

Taking the Fourier transforms of the above two equations, we obtain

$$
f_{0}^{\alpha}=\frac{\partial^{\alpha} f_{0}}{\partial t^{\alpha}}, \quad f_{1}^{\alpha}=\frac{\partial^{\alpha} f_{1}}{\partial t^{\alpha}} .
$$

This completes the proof.

Consider next the $A^{P}$-spaces, for $1 \leq p<\infty$, let

$$
A^{p}(\Gamma)=\left\{f \in L^{1}(\Gamma): \sum_{-\infty}^{\infty}|\hat{f}(n)|^{p}<\infty\right\}
$$

under the norm

$$
\|f\|_{A}{ }_{A}=\|f\|_{1}+\left(\sum_{-\infty}^{\infty}|\hat{f}(n)|^{p}\right)^{1 / p},
$$

$A^{P}(\Gamma)$ forms a Banach space; for the basic properties of $A^{p}$-spaces, see Wang [7].

THEOREM 4. (a) For $f_{0} \in A^{P}\left(\Gamma_{0}\right), f_{1} \in A^{P}\left(\Gamma_{1}\right)$, let

$$
u(t, r)=f_{0} * p_{p}^{0}(t)+f_{1} * p_{r}^{1}(t)
$$

Then $u$ is harmonic on $A$,

$$
\begin{aligned}
& \lim _{r \rightarrow 1} u(t, r)=f_{1}(t) \text { almost everywhere, } \\
& \underset{r \rightarrow r_{0}}{\lim } u(t, r)=f_{0}(t) \text { almost everywhere, } \\
& \|u(t, r)\|_{A} \leq\left\|f_{0}\right\|_{A} p+\left\|f_{1}\right\|_{A} \text { for } r_{0}<r<1 .
\end{aligned}
$$


(b) Let $u(t, r)$ be harmonic on $A$, and $\left\{f_{p}\right\}$ be Cauchy in $L^{1}$-norm as $r \rightarrow 1$ and $r \rightarrow r_{0}$. Assume there is $c>0$, for some $p$, $1 \leq p<\infty$;

$$
\|u(t, r)\|_{A} p \leq c \text { for } r_{0}<r<1 \text {. }
$$

Then

$$
u(t, r)=f_{0} * p_{r}^{0}(t)+f_{1} * p_{r}^{1}(t)
$$

for some $f_{0} \in A^{r}\left(\Gamma_{0}\right), f_{1} \in A^{p}\left(\Gamma_{1}\right)$.

Proof. It suffices to prove (b). By Theorem 2, take $f_{0} \in L^{I}\left(\Gamma_{0}\right)$, $f_{1} \in L^{\perp}\left(\Gamma_{1}\right)$ with

$$
\begin{aligned}
u(t, r) & =f_{0} * p_{r}^{0}(t)+f_{1} * p_{r}^{1}(t), \\
\lim _{t \rightarrow 1} f_{r}(t) & =f_{1}(t) \text { in the } w^{*} \text {-topology, } \\
\lim _{r \rightarrow r_{0}} f_{r}(t) & =f_{0}(t) \text { in the } x^{*} \text {-topology. }
\end{aligned}
$$

Therefore

$$
\begin{aligned}
& \lim _{n \rightarrow 1} \hat{f}_{r}(n)=\hat{f}_{1}(n), \\
& \lim _{n \rightarrow r_{0}} \hat{f}_{r}(n)=\hat{f}_{0}(n),
\end{aligned}
$$

for every integer $n$. Therefore, by the Fatou lemma,

$$
\begin{aligned}
\sum\left|\hat{f}_{1}(n)\right|^{p} & =\sum \lim _{p \rightarrow 1}\left|\hat{f}_{p}(n)\right|^{p} \\
& \leq \frac{\lim }{p \rightarrow 1} \sum\left|\hat{f}_{p}(n)\right|^{p} \\
& \leq c .
\end{aligned}
$$

Similarly $\sum\left|\hat{f}_{0}(n)\right|^{p} \leq c$. 
Thus $f_{0} \in A^{p}\left(\Gamma_{0}\right)$ and $f_{1} \in A^{p}\left(\Gamma_{1}\right)$. This proves the assertion.

In Theorem 4, if $p$ is restricted to lie between 1 and 2 then we can weaken our assumptions.

THEOREM 4'. Let $u(t, r)$ be harmonic on $A$. Assume there is $c>0$, and some $p, 1 \leq p<2$, with

$$
\|u(t, r)\|_{A} p \leq c \text { for } r_{0}<r<1 \text {. }
$$

Then

$$
u(t, r)=f_{0} * p_{p}^{0}(t)+f_{1} * p_{r}^{1}(t)
$$

for some $f_{0} \in A^{P}\left(\Gamma_{0}\right), f_{1} \in A_{p}\left(\Gamma_{1}\right)$.

Proof. By the assumption

$$
\|u(t, r)\|_{A} p \leq c
$$

take $u_{0} \in M\left(\Gamma_{0}\right)$, the measure algebra on $\Gamma_{0}$, and $u_{1} \in M\left(\Gamma_{1}\right)$ such that

$$
u(t, r)=u_{0} * p_{r}^{0}(t)+u_{1} * p_{r}^{1}(t)
$$

Along the same line of the proofs of Theorem 4, we obtain

$$
\begin{aligned}
& \sum\left|\hat{u}_{0}(n)\right|^{p}<\infty, \\
& \sum\left|\hat{u}_{1}(n)\right|^{p}<\infty .
\end{aligned}
$$

By the Hausdorff-Young theorem, $u_{0} \in L^{q}\left(\Gamma_{0}\right), u_{1} \in L^{q}\left(\Gamma_{1}\right)$, where $1 / p+1 / q=1$. Therefore $u_{0} \in L^{1}\left(\Gamma_{0}\right), u_{1} \in L^{1}\left(\Gamma_{1}\right)$ or $u_{0} \in A^{p}\left(\Gamma_{0}\right)$, $u_{1} \in A^{p}\left(\Gamma_{1}\right)$. This completes the proof.

We are now in a position to describe the conjugate Poisson kernel $q(t, r)$ on $A$ and the Hilbert transform $H f$ of $f$ on $\Gamma$. Let

$$
p(t, r)=p_{r}^{0}(t)+p_{r}^{I}(t)
$$


then $p(t, r)=p(t, s)$ where $r s=r_{0}$.

By a theorem of Goldberg [3], $(1-\psi) i \operatorname{sgn}(\xi) \hat{f}(\xi)$ is the Hilbert transform of a function $f$ on the boundary of the strip $\{z \in \mathbb{C}: 0<\operatorname{Im} z<1\}$, where $\psi$ is a testing function with $\psi(0)=1$. Following a well-known result of de Leeuw (see Stein and Weiss [6, p. 260]), there is a multiplier $H$ of $L^{p}(\Gamma)$ to $L^{p}(\Gamma)$ such that

$$
(H f)^{\wedge}(n)=(1-\psi) i \operatorname{sgn}(n) \hat{f}(n)
$$

for all integer $n$ where

$L^{p}(\Gamma)=\left\{f: \Gamma \rightarrow c: f=f_{0}+f_{I}\right.$,

$$
\left.f_{0}(t)=f_{1}(t) \text { almost everywhere, } f_{0} \in I^{p}\left(\Gamma_{0}\right)\right\} \text {. }
$$

We call this $H$ the Hilbert transform of $f$. Note that

$$
\left[(H f) * p_{r}(t)\right]^{\wedge}(m)=\hat{f}(m)(1-\psi) i \operatorname{sgn}(n) \hat{p}_{p}(m)
$$

for all integer $m$. Define

$$
[q(t, r)]^{\wedge}(m)=(1-\psi) i \operatorname{sgn}(n) \hat{p}_{p}(m)
$$

for all integers $m$. Then $q(t, r)$ is harmonic on $A$ with

$$
\begin{aligned}
\|q(t, r)\|_{L} & \leq A\left\|p_{r}\right\|_{L^{p}} \\
& \leq M \quad \text { for } 1<p<\infty .
\end{aligned}
$$

$q(t, r)$ is called the conjugate Poisson kernel of $p(t, r)$.

For $p>0$, define

$$
h^{p}(A)=\left\{(u, v): \sup _{r_{0}<r<1} \int_{0}^{2 \pi}\left(|u(t, r)|^{2}+|v(t, r)|^{2}\right)^{p / 2} d t<\infty\right\}
$$

where $u, v$ are conjugate harmonic functions on $A$, and $u(t, r)=u(t, s)$ with $r s=r_{0}$. Following Fefferman and Ste in [1, p. 174$], v$ can be recovered from $u$ and

$$
\lim _{r \rightarrow 1} u(t, r)=f
$$

exists in the sense of distributions. So $h^{p}(A)$ can be identified with a 
102

Hwai-chiuan Wang

space $h^{p}(\Gamma)$ of distributions on $\Gamma$. In particular, for $p>1$,

$h^{p}(\Gamma)=\left\{f: \Gamma \rightarrow \mathbf{C}: f(t)=f_{0}(t)+f_{1}(t)\right.$ almost everywhere,

$$
\left.f_{0}(t)=f_{I}(t) \text { almost everywhere, } f_{0} \in L^{p}\left(\Gamma_{0}\right)\right\} \text {. }
$$

By Theorem 4' and Stein [5, p. 221], we obtain

PROPOSITION 5. $f \in h^{\perp}(\Gamma)$ if and only if $f \in L^{\perp}(\Gamma), H f \in L^{\perp}(\Gamma)$.

Let $T:(t, r)+r e^{i t}$ where $r_{0} \leq r \leq 1,0 \leq t \leq 2 \pi$.

$$
\begin{aligned}
\Gamma(t)=\left\{\left(t^{\prime}, r\right):\left|t-t^{\prime}\right|<r-r_{0}\right. & \left., r_{0}<r<\frac{1+r_{0}}{2}\right\} \\
& \cup\left\{\left(t^{\prime}, r\right):\left|t-t^{\prime}\right|<1-r, \frac{1+r_{0}}{2}<r<1\right\},
\end{aligned}
$$

$$
\begin{aligned}
\gamma(t) & =T(\Gamma(t)), \\
u^{*}(t) & =\sup _{\left(t^{\prime}, r\right) \in \gamma(t)}\left|u\left(t^{\prime}, r\right)\right| .
\end{aligned}
$$

PROPOSITION 6. If $p>0$ then $u \in h^{p}(A)$ if and only if $u^{*} \in L^{P}\left(\Gamma_{1}\right)$. For each $0<p<\infty$, there is an $N$ so that

$$
B=\left\{\psi \in C^{\infty}(\Gamma):\|\psi\|_{\alpha} \leq 1 \text { for }|\alpha| \leq N\right\}
$$

and if $\psi \in C^{\infty}\left(\Gamma_{1}\right)$ with $\int_{0}^{2 \pi} \psi \neq 0$, then the $L^{p}$-norms of the following functions are equivalent: $u^{*}, \sup _{\left(t^{\prime}, r\right) \in Y(t)}\left|\psi_{r} * f\left(t^{\prime}\right)\right|$,

$\sup _{r_{0}<r<1}\left|\psi_{r} * f(t)\right|$, and $\sup _{r_{0}<r<1} \sup _{\psi \in B}\left|\psi_{r} * f(t)\right|$ where $f \in h^{p}(\Gamma)$ and $u(t, r)=p_{r} \star f(t)$.

PROPOSITION 7. If $\psi \in C^{\infty}\left(\Gamma_{1}\right), \frac{1}{2 \pi} \int_{0}^{2 \pi} \psi=1$ and $\int_{0}^{2 \pi} x^{\alpha} \psi(x) d x=0$ for all $\alpha \neq 0$, then

$$
\|f-\psi * f\|_{H^{p}} \leq c\|f\|_{n} p
$$

https://doi.org/10.1017/S0004972700011515 Published online by Cambridge University Press 
In particular $f \in h^{p} \Rightarrow f-\psi * f \in h^{p}$ where $H^{p}$ is the Hardy space on the woit disk $D$ on the plane.

PROPOSITION 8. The following spaces are identical:

(1) the dual of $h^{1}$;

(2) $\left\{b: b=b_{0}+H b_{1}, b_{0}, b_{1} \in L^{\infty}\right\}$;

(3) $\left\{b \in L^{1}: \sup _{|Q|<1} \frac{1}{|Q|} \int_{Q}\left|b-\frac{1}{|Q|} \int_{Q} b\right|<\infty\right.$, $\sup _{|Q|<1} \frac{I}{|Q|} \int_{Q}|b|<\infty, Q$ cubes on $\left.\Gamma_{1}\right\}$;

(4) $\left\{b \in B M O(D): \psi * b \in L^{\infty}\right\}$ for some $\psi \in C^{\infty}\left(\Gamma_{1}\right)$,

$$
\frac{1}{2 \pi} \int_{0}^{2 \pi} \psi(x) d x=1 \text {, and } \int_{0}^{2 \pi} x^{\alpha} \psi(x) d x=0 \text {. }
$$

Proofs of Propositions 6-8 run parallel to proofs in Goldberg [3].

\section{Some applications}

For $r_{0}, 0<r_{0}<1$, let

$$
\begin{aligned}
& A_{p}=\left\{z \in \mathbb{C}:|z|<r_{0}\right\}, \\
& A_{v}=\left\{z \in \mathbf{C}: r_{0}<|z|<1\right\}, \\
& \Gamma_{0}=\left\{z \in \mathbf{C}:|z|=r_{0}\right\}, \\
& \Gamma_{1}=\{z \in \mathbf{C}:|z|=1\} .
\end{aligned}
$$

Here if we consider $A_{p}$ as the domain filled by a plasma and $A_{v}$ as the domain corresponding to the vacuum, then our results can be applied to solve a special problem in plasma physics.

THEOREM 9. Given two boundary data functions $f_{i} \in L^{p}\left(\Gamma_{i}\right)$, $i=0,1,1<p<\infty$, there is a fronction $u$ defined on the closed unit disk $D=\{z \in \mathbb{C}:|z| \leq 1\}$ such that 


$$
\begin{aligned}
\Delta u & =-\lambda^{2} u \text { on } A_{p}, \\
\Delta u & =0 \quad \text { on } A_{v}, \\
u & =f_{i} \text { on } \Gamma_{i}, \quad i=1,2 .
\end{aligned}
$$

Proof. Take a function $v$ on the closed unit disk $D$ satisfying

$$
\begin{array}{ll}
\Delta v=-\lambda^{2} r_{0}^{2} v & \text { on } \quad\{z \in \mathbb{C}:|z|<1\} \\
v=0 & \text { on }\{z \in \mathbb{C}:|z|=1\}
\end{array}
$$

(see Folland $[2, \mathrm{p} .137]$. Let

$$
w(x)=v\left(x / r_{0}\right)
$$

and $p_{r}^{0}, p_{r}^{1}$ as in Theorem 2. Then

$$
u=w \mathrm{x}_{A_{p}}+\left[f_{0} * p_{r}^{0}+f_{1} * p_{p}^{1}\right] \mathrm{x}_{A_{v}}
$$

is our candidate.

Finally we ask whether our theory can be extended to general domains.

1. If we extend the annulus $A=\left\{z \in \mathbb{C}: r_{0}<|z|<1\right\}$ to a general domain $B$ which is an elliptic annulus possessing two elliptic boundaries, then can our theory apply or not? The affirmative answer is usual for number theory.

2. For $0<r_{1}<1<r_{2}<\infty$, let $\Gamma_{0}=\{z \in \mathbf{C}:|z|=1\}$, $\Gamma_{1}=\left\{z \in \mathbb{C}:|z|=r_{1} \mid, \Gamma_{2}=\left\{z \in \mathbb{C}:|z|=r_{2}\right\}\right.$. Let $C_{1}=\left\{z \in \mathbb{C}: r_{1}<|z|<1\right\}$, and $C_{2}=\left\{z \in \mathbb{C}: 1<|z|<r_{2}\right\}$. Given boundary data functions $f_{i}$ on $\Gamma_{i}, i=0,1,2$, can we apply our theory to solve the generalized Dirichlet problem

$$
\begin{aligned}
\Delta u & =-\lambda^{2} u \text { on } C_{1}, \\
\Delta u & =0 \text { on } C_{2}, \\
u & =f_{i} \text { on } \Gamma_{i} \text { ? }
\end{aligned}
$$




\section{References}

[1] C. Fefferman and E.M. Stein, "HP spaces of several variables", Acta Math. 129 (1972), 137-193.

[2] Gerald B. Folland, Introduction to partial differential equations (Princeton University Press, Princeton, New Jersey, 1976).

[3] David Goldberg, "A local version of real Hardy spaces", Duke Math. J. 46 (1979), 27-42.

[4] Donald Sarason, The $H^{p}$ spaces of con annulua (Memoirs of the American Mathematical Society, 56. American Mathematical Society, Providence, Rhode Island, 1965).

[5] Elias M. Stein, Singular integrals and differentiability properties of functions (Princeton Mathematical Series, 30. Princeton University Press, Princeton, New Jersey, 1970).

[6] Elias M. Stein and Guido Weiss, Introduction to Fourier canalysis on Euclidean spaces (Princeton Mathematical Series, 32. Princeton University Press, Princeton, New Jersey, 1971).

[7] Hwai-Chiuan Wang, Homogeneous Banach algebras (Lecture Notes in Pure and Applied Mathematics, 29. Marcel Dekker, New York, Basel, 1977).

Department of Mathematics, National Tsing Hua University, Hsinchu, Taiwan, Republic of China. 